\title{
3D-FISH analysis reveals chromatid cohesion defect during interphase in Roberts syndrome
}

\author{
Celine Dupont ${ }^{1 *}$, Martine Bucourt ${ }^{2}$, Fabien Guimiot ${ }^{3,4}$, Lilia Kraoua ${ }^{5}$, Daniel Smiljkovski ${ }^{6,7}$, Dominique Le Tessier ${ }^{7}$, \\ Camille Lebugle $^{8}$, Benedicte Gerard ${ }^{5}$, Emmanuel Spaggiari ${ }^{3}$, Pierre Bourdoncle ${ }^{8}$, Anne-Claude Tabet ${ }^{1}$, \\ Brigitte Benzacken ${ }^{1,9}$ and Jean-Michel Dupont ${ }^{6,7}$
}

\begin{abstract}
Background: Roberts syndrome (RBS) is a rare autosomal recessive disorder mainly characterized by growth retardation, limb defects and craniofacial anomalies. Characteristic cytogenetic findings are "railroad track" appearance of chromatids and premature centromere separation in metaphase spreads. Mutations in the ESCO2 (establishment of cohesion 1 homolog 2) gene located in 8p21.1 have been found in several families. ESCO2, a member of the cohesion establishing complex, has a role in the effective cohesion between sister chromatids. In order to analyze sister chromatids topography during interphase, we performed 3D-FISH using pericentromeric heterochromatin probes of chromosomes 1,4,9 and 16, on preserved nuclei from a fetus with RBS carrying compound heterozygous null mutations in the ESCO2 gene.
\end{abstract}

Results: Along with the first observation of an abnormal separation between sister chromatids in heterochromatic regions, we observed a statistically significant change in the intranuclear localization of pericentromeric heterochromatin of chromosome 1 in cells of the fetus compared to normal cells, demonstrating for the first time a modification in the spatial arrangement of chromosome domains during interphase.

Conclusion: We hypothesize that the disorganization of nuclear architecture may result in multiple gene deregulations, either through disruption of DNA cis interaction -such as modification of chromatin loop formation and gene insulation - mediated by cohesin complex, or by relocation of chromosome territories. These changes may modify interactions between the chromatin and the proteins associated with the inner nuclear membrane or the pore complexes. This model offers a link between the molecular defect in cohesion and the complex phenotypic anomalies observed in RBS.

Keywords: Cohesinopathy, ESCO2, Heterochromatin, Limb development

\section{Background}

Roberts Syndrome (RBS, OMIM \#268300) is a rare autosomal recessive disorder first described by John Roberts in 1919. This multiple congenital anomaly syndrome is characterized by cleft lip and palate, nose and ears anomalies, facial hemangioma, hypertelorism, microcephaly, curly silvery blond hair, reductional limb defects leading to oligodactyly or tetraphocomelia, polycystic or dysplastic kidneys, congenital heart defects, enlarged male genitalia, severe growth retardation and intellectual

\footnotetext{
* Correspondence: c.dupont@rdb.aphp.fr

'Unité fonctionnelle de Cytogénétique-Département de Génétique- APHP, Hôpital Robert Debré, 48 Bd Sérurier, 75935 Paris, France

Full list of author information is available at the end of the article
}

deficiency [1]. Vega et al. identified homozygous or compound heterozygous ESCO2 (establishment of cohesion 1 homolog 2) mutations in 15 families with RBS [2]. ESCO2 is one of the two human orthologs of a yeast gene Eco1/Ctf7 involved in sister chromatid cohesion. The C-terminal domain of the protein is evolutionarily conserved and harbors an acetyltransferase (AT) activity [2]. To date, more than 30 different mutations have been described [2-8], most of which result in protein truncation with an absence of evidence for genotype-phenotype correlation [7]. RBS is characterized by a "railroad-track" appearance of metaphase chromosomes, due to premature centromere separation (PCS), a phenomenon also described as heterochromatin repulsion [1]. PCS is more 
prominent in the pericentromeric, heterochromatic regions of chromosomes 1, 9 and 16, in the $\mathrm{p}$ arm of acrocentric chromosomes, and in Yq heterochromatin.

During interphase, chromosomes are organized in the nucleus into individual "chromosome territories" (CTs) that are non-randomly scattered [9]. The position of CT depends on their volume [10] and on gene density [11]. Nuclear architecture and chromatin organization during interphase probably contributes to gene expression [9]. Abnormal spatial organization of the CTs in interphase was reported in tumor cells carrying chromosome translocations [12], in epilepsy [13], in laminopathies [14], and in ICF syndrome $[15,16]$.

To get new insights into the relationship between ESCO2 mutations, cytogenetic anomalies and clinical features in Roberts syndrome, we performed 3D-FISH using pericentromeric heterochromatin $(\mathrm{PH})$ probes of chromosome 1, 4, 9 and 16 on fibroblasts and cytotrophoblasts nuclei of a patient with molecularly defined RBS.

\section{Results}

\section{Patient data}

A 27-year-old mother was referred for cytogenetic prenatal diagnosis after abnormal ultrasound findings (tetraphocomelia, hygroma and facial dysmorphism) at eleven weeks of gestation. Ultrasound scan at 16 weeks confirmed symmetrical limb defects with absent forearms, presence of only one bone in each leg and echogenic bowels. Death occurred in utero at 18 weeks. Parents were healthy, unrelated, from African origin.
The female fetus weighted $70 \mathrm{gr}\left(<<5^{\text {th }}\right.$ centile), and has a crown-rump length of $11.5 \mathrm{~cm}\left(<<5^{\text {th }}\right.$ centile). He had tetraphocomelia (Figure $1 \mathrm{~A}$ ). $\mathrm{X}$ ray survey showed bilateral absence of radii, ulnae, and fibulae, oligodactyly (4 fingers in each hand) and hypoplasia of the fifth toes. He had median frontal bone defect, small, flat nose with hypoplastic nasal bone, hypertelorism, exophthalmia, short philtrum, adhesion of upper lip to the upper gum, and low-set ears. There were no visceral malformations. Histological examination was not contributive. R- and G-banded karyotype from a chorionic villus sample was 46,XX. In skin fibroblasts, C-banded metaphases showed premature centromere separation and puffing of the heterochromatin (Figure 1B).

\section{Mutational analysis of ESCO2}

Analysis of ESCO2 gene in our patient revealed the presence of two compound heterozygous mutations: one mutation is a substitution of an Adenine for a Guanine base $(c .1131+1 G>A)$ and had been previously described [3]; the second mutation is a 4-bp deletion overlapping the end of exon 4 and the beginning of intron 4 (c.954_955+2delAAGT) (Additional file 1: Figure S1). The first mutation (c. $1131+1 \mathrm{G}>\mathrm{A}$ ) is a splice-site mutation in intron 6 resulting in a skipping of exon 6 (r.1014_1131del118) and causing a premature termination of translation (p.R338fs*17) with no evidence of a normal transcript [3]. The second mutation (c.954_955+ 2delAAGT) has never been described before; it interrupts the splice donor site of intron 4 resulting probably in
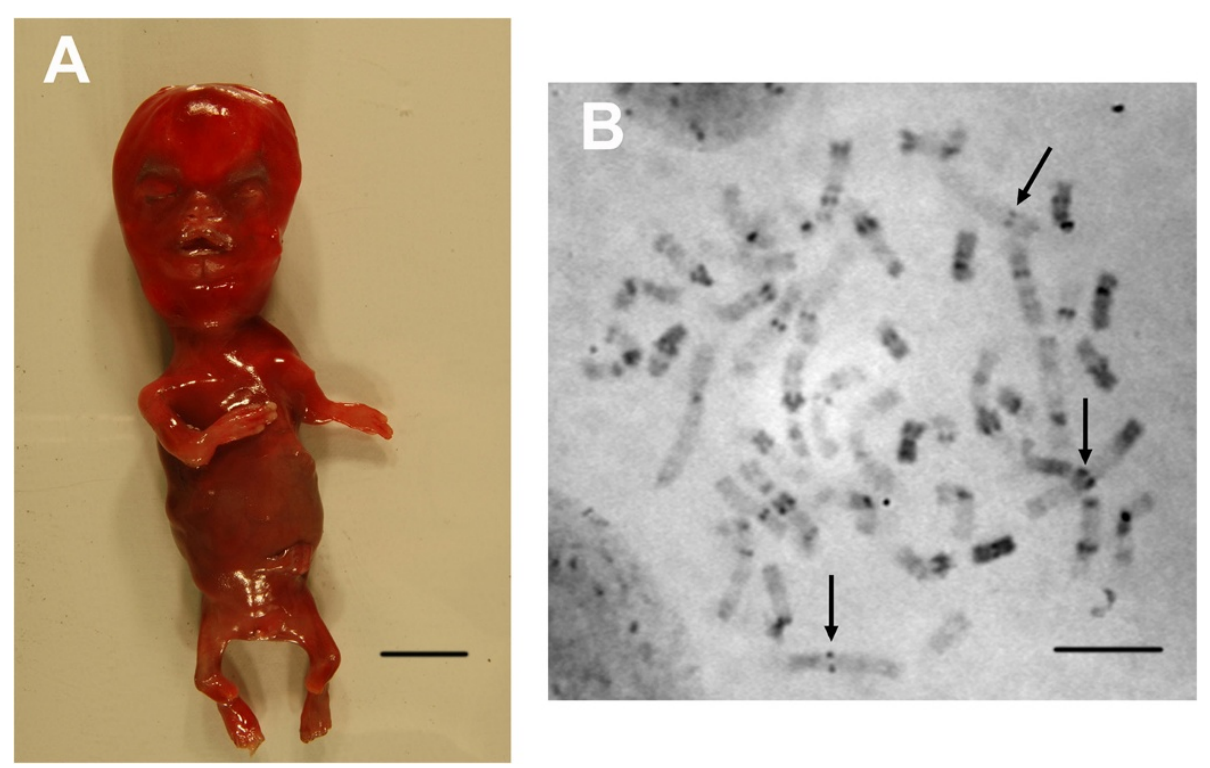

Figure 1 Clinical and cytogenetical description of the fetus. (A): Fetus of 18 weeks gestation with multiple congenital anomalies: hypertelorism, micrognathia, tetraphocomelia and oligodactyly. Scale bar $=2 \mathrm{~cm}$ (B): C-banded metaphase chromosomes from the affected fetus showing the pathognomonic cytogenetic anomaly in Roberts Syndrome: chromosomes with premature centromere separation: PCS (black arrows) and heterochromatin puffing. Scale bar $=5 \mu \mathrm{m}$. 
skipping of exon 4, causing also a premature termination of translation of the ESCO2 protein (p.ASP288Phefs ${ }^{* 22}$ ). Both mutations have to be considered as null alleles, with no functional ESCO2 protein. Unfortunately, the parents were not available for molecular study of ESCO2.

\section{D FISH: Behavior of pericentromeric heterochromatin of chromosome 1 in interphase RBS cells}

Using 3D FISH we showed that approximately $90 \%$ of RBS fibroblasts (7/8) and trophoblasts (23/26) nuclei had a split configuration of one or two PH1 signals (Figure 2A). Pericentromeric heterochromatin of chromosomes 4, 9 and 16 was also split during interphase (Figure 2B). This was not observed in control cells.

In some of the most widely split PH1 sub-territories, a fluorescent signal bridging both chromatids was observed (Figure 2C) that could correspond to intertwined sister DNA molecules.

\section{D FISH: Relocation of PH of chromosome 1 within RBS cells}

Statistical investigations were performed on $\mathrm{PH}$ of chromosome 1 with CB2 probe. Difference in the radial position of PH1 was observed between normal and RBS cytotrophoblasts. Twenty-six RBS nuclei (52 PH1 territories) and 24 control nuclei (48 PH1 territories) were analyzed from cytotrophoblast. The distance of PH1 to the center of the nucleus was significantly increased in RBS nuclei $\left(M^{r} R=0.6700\right) \quad$ compared to controls $\left(M^{r} n=0.5285\right)$ (Figure 3A). This result was interpreted as a localization of PH1 more peripheral in RBS nuclei than in normal cells. There was also a statistically significant difference between normal and RBS cells with respect to mutual PH1 distances (Figure 3B). We observed that in RBS nuclei, both homologous $\mathrm{PH} 1$ territories were closer $\left(\mathrm{M}^{\mathrm{m}} \mathrm{R}=0.3813\right)$ to each other than in normal cells $\left(M^{m} n=0.4527\right)$ (Wilcoxon test). The same analysis was performed on fibroblasts. We were able to analyze 8 RBS nuclei (16 CB2 territories) and 16 control nuclei (31 CB2 territories). No statistically significant difference in the radial position of PH1 was observed between normal and RBS fibroblasts but a general trend towards a more peripheral location of these territories was noticed for RBS fibroblasts $\left(M^{\mathrm{r}} \mathrm{R}=0.6213\right.$ and $\left.\mathrm{M}^{\mathrm{r}} \mathrm{n}=0.5562\right)$.

\section{Discussion}

Genome architecture and 3D folding of chromatin fibers during interphase has been increasingly associated with human diseases through modulation of gene expression [9]. Mutations of genes responsible for this specific

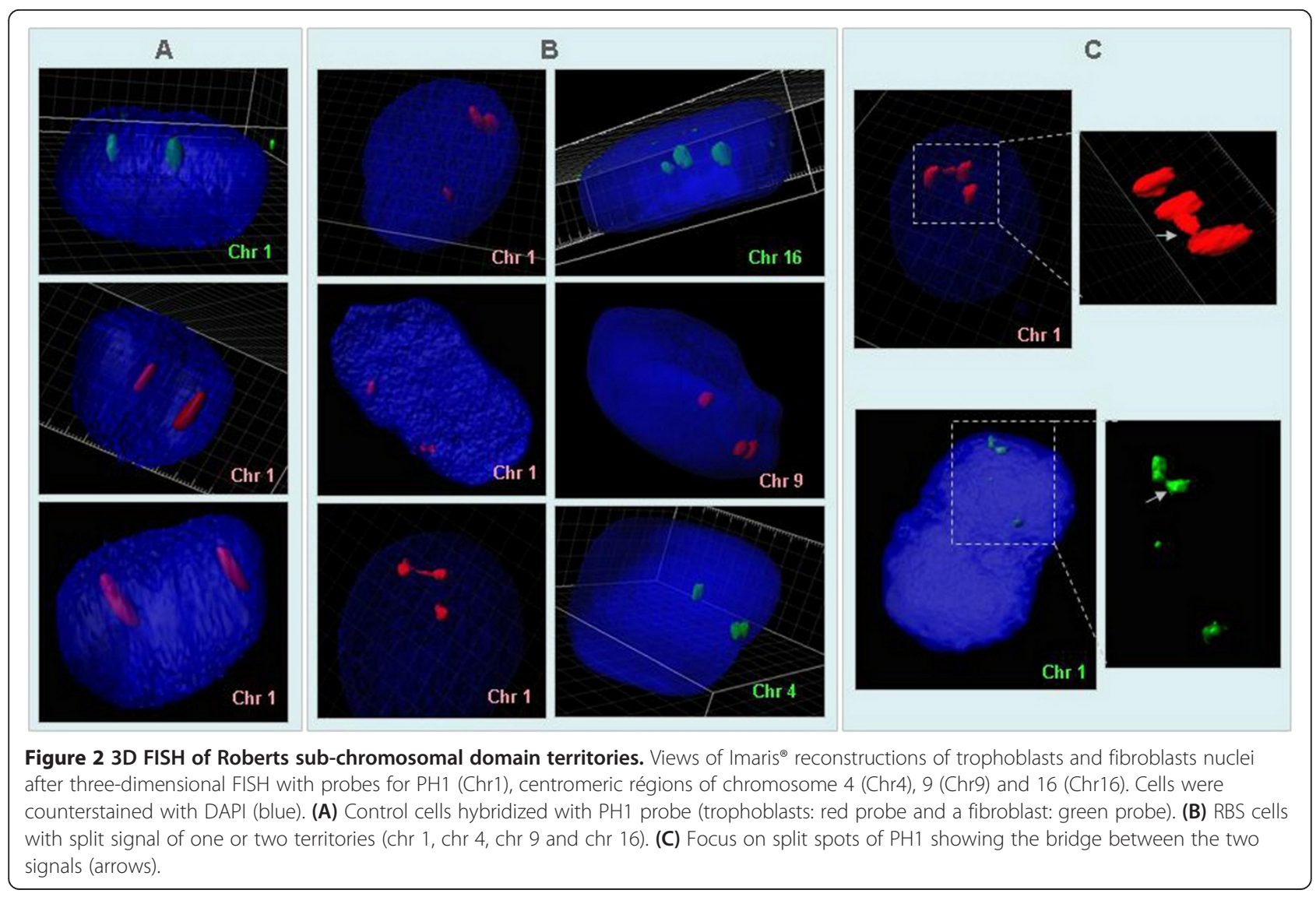




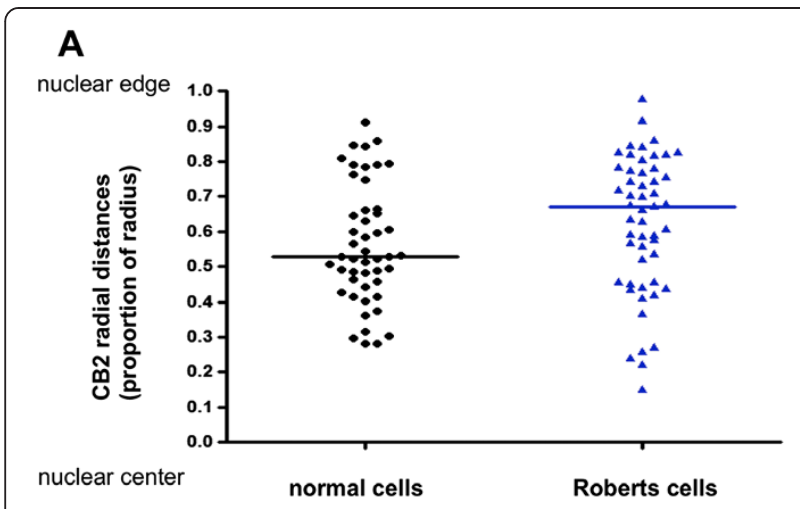

B

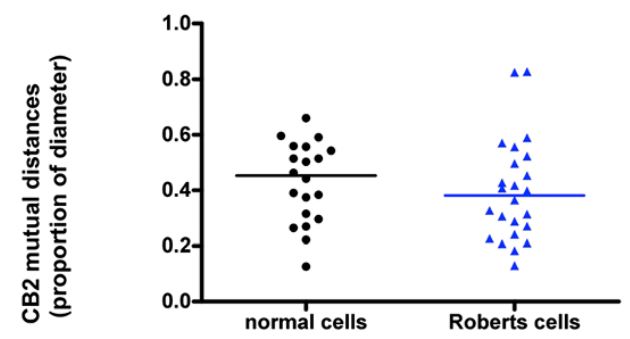

Figure 3 Statistical representation of CB2 radial and mutual positions. (A): Distribution of each $\mathrm{PH} 1$ ( $=\mathrm{CB} 2$ probe) radial position of normal cells (black rings) and Roberts cells (blue triangles). The median was calculated both for normal cells ( $M^{r} n$, black line) and Roberts cells ( $M^{r} R$, blue line). Radial distances are expressed as a proportion of the radius. $M^{r} n=0.5285$ and $M^{r} R=0.6700$ : Wilcoxon test was significant (alpha $=0.05$ ) with a $p$ value of 0.0018 showing a significant relocation of $\mathrm{PH} 1$ territory towards the edge of the nucleus in Roberts cells. (B): Distribution of CB2 mutual position of normal and Roberts cells. The median was calculated both for normal cells ( $M^{m} n$,black line) and Roberts cells ( $M^{m} R$, blue line). Mutual distances are expressed as a proportion of the diameter. $M^{m} n=0.4527$ and $M^{m} R=0.3813$ : Wilcoxon test was significant (alpha $=0.05$ ) with a $p$ value of 0.0829 showing a significant rapprochement of homologous $\mathrm{PH} 1$ territories in Roberts cells.

nuclear organization could lead to developmental disorders such as RBS. A characteristic feature of RBS cells is heterochromatin puffing on metaphase spreads [1]. This finding was the first clue towards a cohesin defect in RBS. Puffing may reflect a modification of the conformation of chromatids. We therefore explored the organization of the centromeric heterochromatin during interphase to check whether modified conformation could reflect an intrinsic reorganization of the chromatin. More than $90 \%$ of RBS nuclei observed in our study showed PH splitting of chromosomes 1, 4, 9 and 16, which was never observed in control nuclei. PCS, which is also seen in metaphase chromosomes in RBS, has never been reported before in interphase nuclei. These new data imply that the underlying conformational anomaly is not confined to metaphase but exists throughout the whole cell cycle. In RBS, loss of ESCO2 acetyltransferase activity is crucial for proper sister chromatid cohesion [6] and most of the ESCO2 mutations described (88\%) lead to a premature stop codon prior or within the acetyltransferase domain which is most likely the case of both mutations found in the reported RBS patient. This loss of ESCO2 function may lead to weaker cohesion between sisters and to the splitting of $\mathrm{PH}$ observed in the nuclei. Moreover it is known that replication of alpha satellite sequences is delayed in RBS cells [17] which could impact chromatin organization, since cohesins are loaded onto DNA during replication. Our 3D-FISH observations favor the handcuff model to explain cohesin-mediated chromosome tethering [18]. In this model, one cohesin ring encircles only one sister chromatid, and both rings are connected by Scc3 and Rad21 subunits to hold sister chromatids together [18]. The cohesion defect could lead to loose juxtaposition of the two chromatids resulting in split FISH signals. Interestingly, many split $\mathrm{PH}$ regions show a bridge between the two signals. This mechanical link might be constituted by inter-twinned centromeric DNA, which has been shown to contribute to centromeric cohesion [19]. Moreover, this bridge could be explained by presence of self-interactions in the $\operatorname{Scc} 1 / \operatorname{Rad} 21$ subunit [18].

There is evidence suggesting that there is a non-random radial nuclear distribution of CTs and chromosomal subregions during interphase. The relative positioning of chromosome domains plays important roles in genome function and gene expression [9]. While the relationship between ESCO2 mutations and the cellular phenotype is well known, the link between the defect in chromatid cohesion and the clinical symptoms of RBS, remains elusive. Because ESCO2 mutations are associated with conformational modifications of chromatin, we explored whether these structural changes could be responsible for phenotypic findings through modification of CT arrangement inside the nucleus. From our 3D-FISH experiments, we could demonstrate a significantly more peripheral position of PH1 in RBS cells compared to control cells.

The pattern of embryonic expression of ESCO2 is consistent with the tissues affected in RBS individuals [7]. A first clue towards the physiopathogenic mechanism of RBS came from the observation of anomalies in cell growth and proliferation. Gordillo and Tomkins [6,20] proposed that defective mitosis and increased cell death might contribute to the reduced growth and developmental phenotype in RBS. Dorsett has suggested that in addition to the proliferation defect, gene expression may be altered in RBS [21]. We hypothesize that abnormal spatial organization of chromosomes domains in interphase nuclei may be the origin, or the consequence of RBS associated chromatin-mediated alterations in gene expression. Previous studies in yeast and drosophila, have established that cohesins play a significant role in gene expression by helping in delimiting the boundaries 
of silenced chromatin domains [22] or by long range activation of homeobox protein genes [23]. Regulation of many genes by cohesin appears to involve the threedimensional (3D) organization of chromatin [24,25], but the basis of this cohesin function remains poorly understood. Investigations in mammals with chromatin immunoprecipitation (ChIP) experiments have revealed that cohesin binds to the same sites as CCCTC binding factor (CTCF) [26]. CTCF is a zinc finger binding transcription factor known to be an enhancer-blocking transcriptional insulator. Compelling evidence that cohesin is required for the function of CTCF has been obtained from the analysis of various loci [27], such as the cytokine IFN- $\gamma, \beta$-globin, apolipoprotein gene cluster, and IGF2/H19 [28]. The stabilization of chromatin loops formed by CTCF depends on cohesin allowing some cis DNA-DNA specific interaction with maternal or paternal alleles [28]. These observations indicate that CTCF and cohesins may control gene expression by mediating local changes in the chromatin conformation, thereby determining which promoters can physically interact with enhancers or other regulatory sequences [28].

For RBS, we suggest that deregulation of cohesin function could promote transcriptional alteration of some developmental genes by the disruption of cis-DNA interactions mediated by CTCF-cohesin complex. Mönnich et al., used a zebrafish model of RBS to analyze, by microarray, the expression of genes downstream of ESCO2 [29]. They showed that Esco2-regulated genes were more likely to be involved in cell-cycle or apoptosis [29]. They proved that even modest Esco2 depletion resulted in strong activation of caspases, in p53/mdm 2 upregulation and in massive cell death, in the first day of development. These results confirm the initial hypothesis on the pathophysiology of RBS. To explore how the deficiency in Esco2 affects cohesin's functions, Whelan et al., generated a mouse harboring a conditional Esco2 allele. Their experiments showed that Esco2 is a real cell viability factor and explained the cellular RBS phenotype by two possible mechanisms: 1) a reduction of Sororin recruitement caused by reduced Smc3 acetylation in mutants and resulting in inefficient pericentromeric cohesion at $\mathrm{S}$ phase- 2) a relocalisation of Shugoshin (Sgo1) to the chromosome arm resulting in a deprotection of centromeric cohesion [30].

The expression of some key genes is probably modified in tissues from RBS patients and could be measured by microarray studies in order to explore how this modification in the spatial arrangement of chromosome affects the transcriptome. We observed a change in spatial conformation of $\mathrm{PH} 1$ and there are some genes on chromosome 1, whose deregulation could lead to the RBS phenotype. Indeed, there are genes that play a role in cell growth and division (proliferation) as CDC73 (1q25), LEPRE1 (1p34.1), NRAS (1p13.2) and ORC1 (1p32). Some others have an important role in the growth and development of bones: ALPL (1p36.12), COL9A2 (1p33p32), COL11A1 (1p21), SKI (1p36.33) and TGFB2 (1q41). KIF1 (1p36.2) is involved in programmed cell death (apoptosis). Finally ARID1A (1p35.3) and LBR (1q42.1) regulate gene activity by chromatin remodeling and $R B M 8$ (1q21.1) gene is involved in TAR syndrome. All these genes and probably others on different chromosomes could be differentially expressed secondary to the modification in PH1 location or cohesin dysfunction in RBS cells and thus result in abnormal phenotype. Unfortunately, expression analysis by qPCR or expression microarray studies, which requires fresh fetal tissues, could not be performed in this RBS patient.

Because the main silent chromatin domains localize to the nuclear border, the nuclear periphery is generally viewed as an area of "gene silencing". Silencing is mediated by the tethering of chromatin to the lamina, which provides a distinct regulatory environment through laminaassociated protein and chromatin structure modification [31]. However, the nuclear border is also associated with specific transcriptional activation [32]. Interestingly, the laminar interacting domains are flanked by insulator protein-binding sites like CTCF [33]. We hypothesize that PH1 relocation observed in RBS cells could promote a modification in chromatin-lamina interactions secondary to an altered cohesin function. At this point, we cannot determine whether $\mathrm{PH}$ delocalization is a direct consequence of ESCO2 dysfunction, or whether absence of ESCO2, by an unknown mechanism, modifies gene expression, resulting in $\mathrm{PH}$ relocalization. $\mathrm{PH}$ relocation could be responsible for further alterations in transcriptional activities that finally would explain the RBS phenotype.

\section{Conclusion}

In conclusion, we have shown that ESCO2 mutations lead to a reorganization of the topology of interphase nucleus, opening new perspectives to enlighten the physiopathology of cohesinopathies.

\section{Methods}

Written informed consent was obtained from the patient for publication of this study and any accompanying images. A copy of the written consent is available for review by the Editor-in-Chief of this journal.

\section{Molecular analysis of ESCO2}

Genomic DNA was isolated from fetal skin sample using the QIAamp DNA Blood Midi kit (QIAGEN GmbH, Hilden, Germany) following the manufacturer's recommendations. The coding sequences and intron-exon junctions of ESCO2 (GenBank NM_001017420.2) were directly sequenced. Purified PCR amplification products 
were sequenced using the BigDye Terminator Cycle Sequencing Kit v.1.1 (Applied Biosystems, Foster City, California, USA) according to the manufacturer's instructions, and were resolved on an ABI 3130xl automated sequencer (Applied Biosystems). Sequence data were aligned with SeqScape 2.0 software and compared to the published sequences of ESCO2.

\section{Cell culture and preparation}

Chorionic villus sampling was performed at 11 WG. Fibroblasts were obtained from Achilles tendon during fetal necropsy. Cytotrophoblasts and fibroblasts cultures were set up following standard protocols. Cells were then washed and stored in PBS. Control cytotrophoblasts and fibroblasts were obtained from two cytogenetically normal fetuses.

\section{D FISH}

The protocol was derived from Dupont et al. [16]. Airdrying of the preparation was carefully avoided in all experiments to preserve the 3D structure of nuclei. In-house PCR-amplified probe for pericentromeric heterochromatin of chromosome 1 (PH1 = CB2:1q12, satellite II) was labelled with biotin or digoxygenin-16-dUTP by PCR amplification while heterochromatin probes for human chromosome 4 ( $\mathrm{PH} 4=\mathrm{D} 4 \mathrm{Z1}$, $\alpha$ satellite), chromosome 9 (PH9= D9Z3, satellite III) and chromosome 16 $(\mathrm{PH} 16=\mathrm{D} 16 \mathrm{Z2}$, asatellite $)$ were FITC or rhodamin labelled (probes provided by Cytocell ${ }^{\circ}$, Cambridge, UK). Hybridization was carried out at $37^{\circ} \mathrm{C}$ in a humidified chamber for approximately 14 hours. After washing, following standard procedures, digoxigenated and biotinylated probes were detected by anti-digoxigenin-rhodamine or by avidin-FITC conjugate, respectively. All slides were counterstained with $1 \mu \mathrm{g} / \mathrm{ml}$ DAPI.

\section{Image acquisition and microscopy}

$3 \mathrm{D}$ preparations were examined using a Leica ${ }^{\oplus}$ (Leica microsystem GmbH, Wetzlar, Germany) (TCS SP2 AOBS) confocal microscope equipped with a $63 x$ objective. The camera and the microscope were controlled by the Leica ${ }^{\circ}$ Confocal Software (LCS). Stacks of optical sections were collected from nuclei showing apparently complete and specific hybridization signals in all channels. Stacks were obtained with an image size of $512 \times 512$ pixels. The focus step between sections was $0.5 \mu \mathrm{m}$ on the average.

For chromosome 1 we analyzed 26 cytotrophoblasts (52 territories) and 8 fibroblasts (16 territories) nuclei from RBS patient. For chromosomes 4, 9 and 16 we only have few nuclei (5 for each probe) to observe and we chose to focus on chromosome 1 to draw statistic data. In parallel, we analyzed 24 cytotrophoblasts (48 territories) and 15 fibroblasts (30 territories) nuclei from a control patient, only for chromosome 1 .
Roberts's cellular phenotype is mainly defined by an abnormal morphology of pericentromeric constitutive heterochromatin, including the large $\mathrm{PH} 1$ domain on chromosome 1. This region was selected for further analysis because a specific PCR probe was available in the laboratory [16] and because chromosome 1 was shown to adopt a non ambiguous peripheral position in fibroblast nuclei [10].

\section{Quantitative evaluation and statistical analysis of data}

The 3D reconstructions of PH1-, PH4-, PH9- and PH16labelled nuclei captured by confocal microscopy were performed using the IMARIS ${ }^{\oplus} 7.1$ software (Biplane Scientific Software, St. Paul, MN). To calculate distances in an ellipsoid object, the 3D coordinates for each fluorescent object (the nucleus or the $\mathrm{PH}$ fluorescent signals) were determined by IMARIS ${ }^{\oplus}$. The radial position of PH1 ([nuclear center]-to-[PH1] distances) and mutual distances between the two PH1 territories ([PH1]-[PH1] distances) were calculated using a MATLAB ${ }^{\odot}$ (MathWorks, Natick, MA) script. The measured radial positions of PH1 were normalized to the nuclear radius to allow accurate length comparison. They are presented as a percentage of the nuclear radius [\% of $\mathrm{R}]$. In the same way, mutual distances were normalized to the largest nuclear diameter. Statistical analysis of distances was carried out using GraphPad Prism $^{\odot}$ (GraphPad, La Jolla, CA), using a non-parametric Wilcoxon two-sample rank-sum test comparing median differences in normal and RBS cells. The median was first calculated for normal cells $\left(M^{r} n\right.$ for radial and $M^{m} n$ for mutual distances) and then for RBS cells $\left(M^{r} R\right.$ for radial and $\mathrm{M}^{\mathrm{m}} \mathrm{R}$ for mutual distances).

\section{Additional file}

Additional file 1: Figure S1. Chromatograms of the sequencing results. Chromatograms of the sequencing results of ESCO2 gene showing both mutations: (A) The mutation (c.954_955 + 2delAAGT): a 4-bp deletion overlapping the end of exon 4 and the beginning of intron 4. (B) The mutation $(c .1131+1 \mathrm{G}>\mathrm{A})$ : a substitution of an Adenine for a Guanine base, a splice-site mutation in intron 6 .

\section{Competing interests}

The authors declare that they have no competing interests.

\section{Authors' contributions}

$C D$, carried out all the experiments of this study except mutational analysis of ESCO2 and drafted the manuscript. MB, performed the autopsy of the fetus. FG, participated in the 3D-FISH images analysis, performed the statistical analysis, and helped to draft the manuscript. LK, performed the mutational analysis of ESCO2. DS, participated in the images acquisition using confocal microscopy. DLT, participated in the 3D-FISH experiments. CL, designed the math software for calculating intranuclear radial and mutual positions from 3D images. BG, performed the mutational analysis of ESCO2. ES participated in the statistical analysis. $\mathrm{PB}$, participated in the images acquisition using confocal microscopy. $A C T$, helped to draft the manuscript. $\mathrm{BB}$, helped to draft the manuscript. JMD, conceived the study, and participated in its design and coordination and helped to draft the manuscript. All authors read and approved the final manuscript. 


\section{Author details}

'Unité fonctionnelle de Cytogénétique-Département de Génétique- APHP, Hôpital Robert Debré, 48 Bd Sérurier, 75935 Paris, France. ${ }^{2}$ Laboratoire de Fœetopathologie- APHP, Hôpital Jean Verdier, Bondy, France. ${ }^{3}$ Service de Biologie du Développement- APHP, Hôpital Robert Debré, Paris, France. ${ }^{4}$ Université Paris Diderot Sorbonne Paris Cité, UMR 1141, F-75019 Paris, France. ${ }^{5}$ Unité fonctionnelle de Génétique moléculaire - Département de Génétique- APHP, Hôpital Robert Debré, Paris, France. ${ }^{6}$ Génomique, Epigénétique et Physiopathologie de la Reproduction, U1016 INSERM-UMR 8104 CNRS (Institut Cochin), Université Paris Descartes, Faculté de Médecine, Paris, France. 'Laboratoire de Cytogénétique- APHP, Hôpitaux Universitaires Paris Centre, Paris, France. ${ }^{8}$ Institut Cochin, Plateforme d'imagerie cellulaire, Paris, France. ${ }^{9}$ Service d'Histologie, Embryologie et Cytogénétique, Biologie de la Reproduction- APHP, Hôpital Jean Verdier, Bondy, France; UFR-SMBH, Paris XIII, France.

Received: 11 June 2014 Accepted: 21 August 2014

Published online: 30 September 2014

\section{References}

1. Van Den Berg DJ, Francke U: Roberts syndrome: a review of 100 cases and a new rating system for severity. Am J Med Genet 1993 47:1104-1123.

2. Vega H, Waisfisz Q, Gordillo M, Sakai N, Yanagihara I, Yamada M, van Gosliga D, Kayserili H, Xu C, Ozono K, Jabs EW, Inui K, Joenje H: Roberts syndrome is caused by mutations in ESCO2, a human homolog of yeast ECO1 that is essential for the establishment of sister chromatid cohesion. Nat Genet 2005, 37:468-470.

3. Schule B, Oviedo A, Johnston K, Pai S, Francke U: Inactivating mutations in ESCO2 cause SC phocomelia and Roberts syndrome: no phenotypegenotype correlation. Am J Hum Genet 2005, 77:1117-1128.

4. Resta N, Susca FC, Di Giacomo MC, Stella A, Bukvic N, Bagnulo R, Simone C, Guanti G: A homozygous frameshift mutation in the ESCO2 gene: evidence of intertissue and interindividual variation in Nmd efficiency. J Cell Physiol 2006, 209:67-73.

5. Schulz S, Gerloff C, Ledig S, Langer D, Volleth M, Shirneshan K, Wieacker P: Prenatal diagnosis of Roberts syndrome and detection of an ESCO2 frameshift mutation in a Pakistani family. Prenat Diagn 2008, 28:42-45.

6. Gordillo M, Vega H, Trainer AH, Hou F, Sakai N, Luque R, Kayserili H, Basaran S, Skovby F, Hennekam RC, Uzielli ML, Schnur RE, Manouvrier S, Chang S, Blair E, Hurst JA, Forzano F, Meins M, Simola KO, Raas-Rothschild A, Schultz RA, McDaniel LD, Ozono K, Inui K, Zou H, Jabs EW: The molecular mechanism underlying Roberts syndrome involves loss of ESCO2 acetyltransferase activity. Hum Mol Genet 2008, 17:2172-2180.

7. Vega H, Trainer AH, Gordillo M, Crosier M, Kayserili H, Skovby F, Uzielli ML, Schnur RE, Manouvrier S, Blair E, Hurst JA, Forzano F, Meins M, Simola KO, Raas-Rothschild A, Hennekam RC, Jabs EW: Phenotypic variability in 49 cases of ESCO2 mutations, including novel missense and codon deletion in the acetyltransferase domain, correlates with ESCO2 expression and establishes the clinical criteria for Roberts syndrome. J Med Genet 2010, 47:30-37.

8. Goh ES, Li C, Horsburgh S, Kasai Y, Kolomietz E, Morel CF: The Roberts syndrome/SC phocomelia spectrum-a case report of an adult with review of the literature. Am J Med Genet A 2010, 152A:472-478.

9. Cremer T, Cremer M: Chromosome territories. Cold Spring Harb Perspect Biol 2010, 2:a003889.

10. Bolzer A, Kreth G, Solovei I, Koehler D, Saracoglu K, Fauth C, Müller S, Eils R, Cremer C, Speicher MR, Cremer T: Three-dimensional maps of all chromosomes in human male fibroblast nuclei and prometaphase rosettes. PLOS Biol 2005, 3:e157

11. Croft JA, Bridger JM, Boyle S, Perry P, Teague P, Bickmore WA: Differences in the Localization and Morphology of Chromosomes in the Human Nucleus. J Cell Biol 1999, 145:1119-1131.

12. Bickmore WA, Teague P: Influences of chromosome size, gene density and nuclear position on the frequency of constitutional translocations in the human population. Chromosome Res 2002, 10:707-715.

13. Borden J, Manuelidis L: Movement of the $\mathrm{X}$ chromosome in epilepsy. Science 1988, 242:1687-1691.

14. Meaburn KJ, Cabuy E, Bonne G, Levy N, Morris GE, Novelli G, Kill IR, Bridger JM: Primary laminopathy fibroblasts display altered genome organization and apoptosis. Aging Cell 2007, 6:139-153.
15. Matarazzo MR, Boyle S, D'Esposito M, Bickmore WA: Chromosome territory reorganization in a human disease with altered DNA methylation. Proc Natl Acad Sci U S A 2007, 104:16546-16551.

16. Dupont C, Guimiot F, Perrin L, Marey I, Smiljkovski D, Le Tessier D, Lebugle C, Baumann C, Bourdoncle P, Tabet AC, Aboura A, Benzacken B, Dupont JM: $3 \mathrm{D}$ position of pericentromeric heterochromatin within the nucleus of a patient with ICF syndrome. Clin Genet 2012, 82:187-192.

17. Barbosa AC, Otto PA, Vianna-Morgante AM: Replication timing of homologous alpha-satellite DNA in Roberts syndrome. Chromosome Res 2000, 8:645-650.

18. Zhang N, Kuznetsov SG, Sharan SK, Li K, Rao PH, Pati D: A handcuff model for the cohesin complex. J Cell Biol 2008, 183:1019-1031.

19. Díaz-Martínez LA, Giménez-Abián JF, Clarke DJ: Cohesin is dispensable for centromere cohesion in human cells. PLoS One 2007, 2:e318.

20. Tomkins DJ, Sisken JE: Abnormalities in the cell-division cycle in Roberts syndrome fibroblasts: a cellular basis for the phenotypic characteristics? Am J Hum Genet 1984, 36:1332-1340.

21. Dorsett D: Roles of the sister chromatid cohesion apparatus in gene expression, development, and human syndromes. Chromosoma 2007, 116:1-13.

22. Donze D, Adams CR, Rine J, Kamakaka RT: The boundaries of the silenced HMR domain in Saccharomyces cerevisiae. Genes Dev 1999, 13:698-708.

23. Rollins RA, Morcillo P, Dorsett D: Nipped-B, a Drosophila homologue of chromosomal adherins, participates in activation by remote enhancers in the cut and Ultrabithorax genes. Genetics 1999, 152:577-593.

24. Dorsett D: Cohesin: genomic insights into controlling gene transcription and development. Curr Opin Genet Dev 2011, 21:199-206.

25. Horsfield JA, Print CG, Mönnich M: Diverse developmental disorders from the one ring: distinct molecular pathways underlie the cohesinopathies. Front Genet 2012, 3:171.

26. Wendt KS, Yoshida K, Itoh T, Bando M, Koch B, Schirghuber E, Tsutsumi S, Nagae G, Ishihara K, Mishiro T, Yahata K, Imamoto F, Aburatani H, Nakao M Imamoto N, Maeshima K, Shirahige K, Peters JM: Cohesin mediates transcriptional insulation by CCCTC-binding factor. Nature 2008, 451:796-801.

27. Wendt KS, Peters JM: How cohesin and CTCF cooperate in regulating gene expression. Chromosome Res 2009, 17:201-214.

28. Bose T, Gerton JL: Cohesinopathies, gene expression, and chromatin organization. J Cell Biol 2010, 189:201-210.

29. Mönnich M, Kuriger Z, Print CG, Horsfield JA: A zebrafish model of Roberts syndrome reveals that Esco2 depletion interferes with development by disrupting the cell cycle. PLoS One 2011, 6:e20051.

30. Whelan G, Kreidl E, Peters JM, Eichele G: The non-redundant function of cohesin acetyltransferase Esco2: some answers and new questions. Nucleus 2012, 3:330-334.

31. Deniaud E, Bickmore WA: Transcription and the nuclear periphery: edge of darkness? Curr Opin Genet Dev 2009, 19:187-191.

32. Taddei A, Van Houwe G, Hediger F, Kalck V, Cubizolles F, Schober H, Gasse SM: Nuclear pore association confers optimal expression levels for an inducible yeast gene. Nature 2006, 441:774-778

33. Mekhail K, Moazed D: The nuclear envelope in genome organization expression and stability. Nat Rev Mol Cell Biol 2010, 11:317-328.

\section{doi:10.1186/s13039-014-0059-6}

Cite this article as: Dupont et al:: 3D-FISH analysis reveals chromatid cohesion defect during interphase in Roberts syndrome. Molecular Cytogenetics 2014 7:59. 